\title{
Performance Analysis of Multirate Multiservice Optical CDMA Networks Adopting Overlapping PPM Signaling
}

\author{
Ahmed E. Farghal, Hossam M. H. Shalaby, Senior Member, IEEE, and Zen Kawasaki, Senior Member, IEEE
}

\begin{abstract}
In this paper, an optical code-division multiple-access (OCDMA) system employing overlapping pulse-position modulation (OPPM) is proposed to support multimedia services with different bit-rate and quality-of-service $(\mathrm{QoS})$ requirements in OCDMA networks. Both wrapped OPPM (WOPPM) and unwrapped OPPM (UOPPM) are considered. The proposed system achieves multirate and multi-QoS transmission by using multilength variable-weight optical orthogonal codes (MLVW-OOCs) as signature sequences and using different values of OPPM modulation parameters. Furthermore, numerical analysis is performed for two different receiver structures, namely, correlation receivers with and without hard-limiters. In our analysis, the multiple-access interference (MAI) is considered as the main performance limiting factor. In addition, the performance of the proposed system is evaluated and compared to that of traditional OOK-OCDMA system. Our results reveal that under both pulsewidth and throughput constraints, the performances of the proposed WOPPM- and UOPPM-OCDMA systems are significantly superior to that of OOK-OCDMA system.
\end{abstract}

Index Terms-Bit-error-rate (BER), code division multiple access (CDMA), multiple-access interference (MAI), ON-OFF keying (OOK), overlapping pulse-position modulation (OPPM), quality of service $(\mathbf{Q o S})$.

\section{INTRODUCTION}

O PTICAL code-division multiple-access (OCDMA) has gained much attention as one of the promising multipleaccess technique for future optical-access networks [1]-[4]. This is mainly due to the attractive OCDMA features, such as full asynchronous entire optical channel sharing among multiple users without any waiting time, which results in low latency access and less transmission delay. In addition to simplified and distributed network control, secure data transmission, soft capacity on demand, bursty traffic, and the ability to support multimedia services differentiation at the physical layer [5], [6].

Both on-off keying (OOK) and pulse-position modulation (PPM) have been commonly used as modulation techniques in

Manuscript received February 24, 2014; revised June 10, 2014; accepted June 16, 2014. Date of publication June 17, 2014; date of current version July 19, 2014. This work was supported by the Egyptian Ministry of Higher Education.

A. E. Farghal is with the Department of Electronics and Communications Engineering, Egypt-Japan University of Science and Technology, Alexandria 21934, Egypt (e-mail: ahmed.farghal@ejust.edu.eg).

H. M. H. Shalaby is with the Department of Electronics and Communications Engineering, Egypt-Japan University of Science and Technology, Alexandria 21934, Egypt, on leave from the Electrical Engineering Department, Alexandria University, Alexandria 21544, Egypt (e-mail: shalaby@ieee.org).

Z. Kawasaki is with the Graduate School of Engineering, Osaka University, Osaka 565-0871, Japan (e-mail: zen@comm.eng.osaka-u.ac.jp).

Color versions of one or more of the figures in this paper are available online at http://ieeexplore.ieee.org.

Digital Object Identifier 10.1109/JLT.2014.2331700
OCDMA systems [7]-[9]. If the average power rather than optical pulsewidth is the constraining factor, PPM-OCDMA technique performs better than OOK-OCDMA technique. Under fixed throughput and pulsewidth constraints, replacing OOK by PPM produces no advantage in OCDMA systems. On the other hand, OPPM (with its two variants, wrapped OPPM (WOPPM) and unwrapped OPPM (UOPPM)), maintains the advantages of PPM schemes over OOK schemes in terms of power efficiency and implementation simplicity [10], [11]. When compared to the PPM-OCDMA systems, OPPM-OCDMA techniques achieve higher tolerance to MAI, which results in improvement of both BER and optical channel capacity, without the need to decrease the light pulsewidth.

Future optical communications networks are expected to provide broadband access to a high number of users for various multimedia services (e.g., data, image, voice, and video), where supporting various kinds of data (with very different transmission rates and QoS traffic requirements) is becoming very essential. The conventional OCDMA systems cannot support such kinds of data, because a code with the same parameters (code length and weight) is assigned to each user and thus all users have equal data rate and equal error performance. Various techniques have been proposed for the support of multirate services provision in OOK-OCDMA networks. Multicode and multi-length codes are two popular techniques employed to provide multi-rate service in OOK-OCDMA networks [3], [12]-[14]. On the other hand, variable-weight optical codes (with identical-chip-power) and multilevel (different-chip-power) signaling have been introduced to provide QoS differentiation in OCDMA networks by controlling the BER at the receiver [15]-[22]. In [23], multirate transmission is achieved using adaptive OPPM in OCDMA networks by accommodating users with different number of symbols $M$. In addition, optical power controller is employed to provide variable QoS levels for different traffic types. In [24], differentiated QoS for different service-classes is provided by using multilevel OCDMA signaling technique based on WOPPM.

In this paper we propose an OPPM scheme to support multimedia services with different transmission rates and QoS requirements in OCDMA networks. The idea is based on varying the OPPM modulation parameters and utilizing multi-length variable-weight optical orthogonal codes (MLVW-OOCs) as the signature sequences. The length and weight of OOCs and OPPM parameters are designed based on the characteristics of the supported service classes under constraints on both the optical pulsewidth and throughput among different classes. Furthermore, the case of frame length constraint is also considered. 


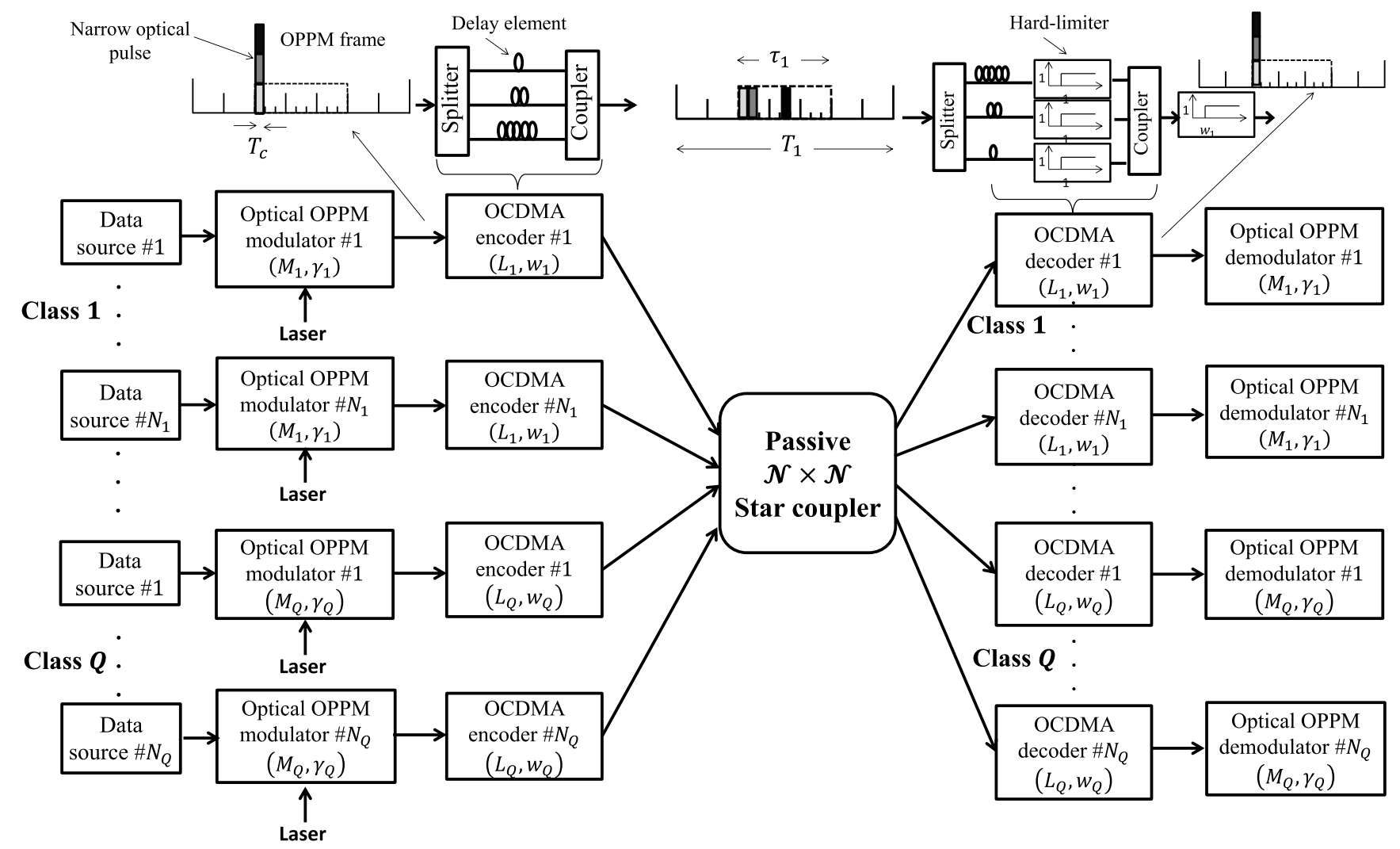

Fig. 1. Model of a multirate multi-QoS OPPM-OCDMA system.

Furthermore, we analyze the performance of the proposed system and derive upper bounds on the probabilities of error for correlation receivers with and without hard-limiters, taking into account the effect of MAI. Our analyses are performed for both WOPPM and UOPPM modulation techniques. Next, we numerically compare the performance of the proposed systems with that of MLVW-OOC OOK systems. Our results reveal that under pulsewidth and throughput constraints, the performance of the proposed W/UOPPM-OCDMA system is better than that adopting OOK-OCDMA scheme.

The rest of the paper is organized as follows. Our proposed multirate multi-QoS model of OCDMA systems adopting OPPM signaling is presented in Section II. Section III is devoted to the performance analysis of the proposed systems. Numerical results are presented in Section IV. Finally, the paper is concluded in Section V.

\section{MultiRate Multi-QoS OPPM-OCDMA System Model}

We denote the number of classes by $Q \geq 1$ and denote the set of classes by $\Omega \stackrel{\text { def }}{=}\{1,2, \ldots, Q\}$. Thus, each service class $k \in \Omega$ can be characterized by $\left(L_{k}, w_{k}, M_{k}, \gamma_{k}\right)$, where $L_{k}, w_{k}, M_{k}$, and $\gamma_{k}$ denote the code length, code weight, number of symbols, and overlapping index in class $k$, respectively. The general model for a multirate multi-QoS OPPM-OCDMA system is shown in Fig. 1. In this figure, we assume that there are $N_{k}$ simultaneous users in class $k \in \Omega$. The total number of simultaneous users in the network $\mathcal{N}$ is thus:

$$
\mathcal{N}=N_{1}+N_{2}+\cdots+N_{Q} .
$$

Moreover, the numbers of available codewords in the specified classes are subject to the following constraint [16]:

$$
\frac{\sum_{k=1}^{Q} N_{k} \times w_{k} \times\left(w_{k}-1\right)}{L_{\max }-1} \leq 1
$$

where $L_{\max }$ is the maximum code length of the $Q$ classes.

\section{A. Transmitter Side}

The transmitter of the $i$ th user in service class $k \in \Omega$ transmits $M_{k}$-ary data symbols $b_{k i} \in \mathcal{M}_{k}$ continuously and asynchronously, where the discrete set $\mathcal{M}_{k} \stackrel{\text { def }}{=}\left\{0,1, \ldots, M_{k}-1\right\}$. The input symbol stream of the $i$ th user in class $k$ is passed to the corresponding optical OPPM modulator, where a narrow optical pulse with duration $T_{c}$, called chip time, is generated and time delayed depending on the incoming data symbol. This optical pulse occupy the first chip time of one of the $M_{k}$ spreading intervals (called slots) of duration $\tau_{k}$. Next, the OCDMA encoder corresponding to the $i$ th user in class $k \in \Omega$ maps its data into the $i$ th code, $c_{i}^{k}$, from $C_{k}=\left\{c_{1}^{k}, c_{2}^{k}, \ldots, c_{N_{k}}^{k}\right\}$ code set available in class $k$ with weight $w_{k}$, length $L_{k}$, and duration $\tau_{k}=L_{k} T_{c}$, located in one of the $M_{k}$ time slots within a frame $T_{k}$. Here, we employ OOCs, with out-of-phase autocorrelation and maximum cross-correlation bounded by one, as our set of signature code sequences [25]. Finally, all spreading signals from all users 


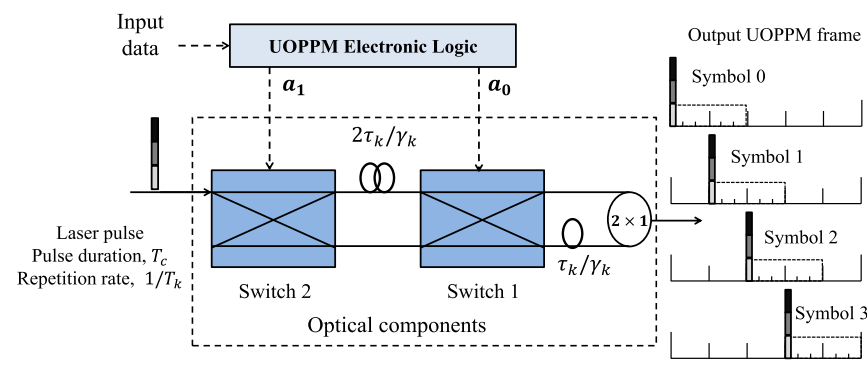

Fig. 2. UOPPM modulator for $M_{k}=4$, and $\gamma_{k}=2$.

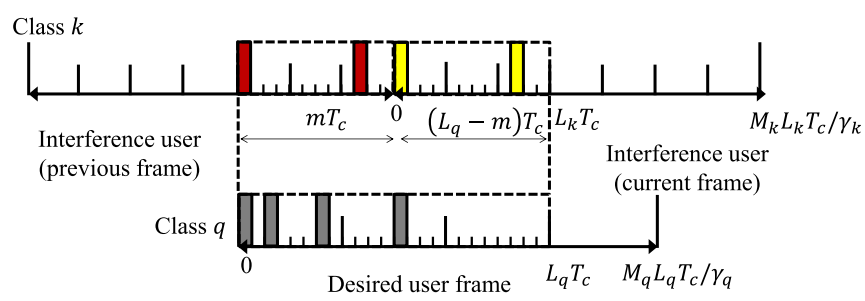

(a)

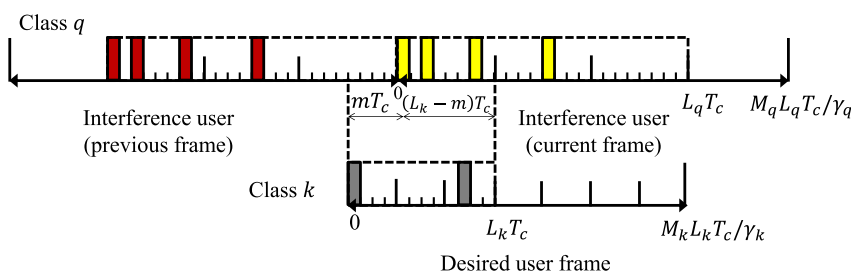

(b)

Fig. 3. Interference contribution: (a) Desired low-rate user interfered with two frames of an undesired high-rate chip-synchronous user. (b) Desired high-rate user interfered with two frames of an undesired low-rate chip-synchronous user.

in all classes are combined together to form one optical signal which is transmitted across the passive $\mathcal{N} \times \mathcal{N}$ star coupler to all receivers.

\section{B. Receiver Side}

At the receiver side, for each service-class $k$, the OCDMA decoders of the designed $N_{k}$ codewords on that class are implemented and the data is detected by correlating the received optical signal from all $\mathcal{N}$ users with the signature sequence that characterizes the desired user and then converted (using a photodetector) into an electrical signal which is passed to the OPPM demodulator to decide on the data [11].

\section{OPPM Technique}

In OPPM technique, a slot of duration $\tau_{k}=L_{k} T_{c}$ is divided into $\gamma_{k} \in\left\{1,2, \ldots, M_{k}-1\right\}$ subslots of width $\tau_{k} / \gamma_{k}$ each. Each slot is forced to overlap with its adjacent slots so that there is a $\tau_{k} / \gamma_{k}$ delay between any two adjacent slots. In this work, we consider two modulation formats for OPPM-OCDMA systems, namely, wrapped and unwrapped. In WOPPM if there is no enough space to accommodate a complete spreading interval a cyclic shift is allowed to occur and the remaining part of the spreading interval is wrapped to the beginning of the frame. On the other hand, there are no such cyclic shifts in UOPPM. The relation between $T_{k}, M_{k}, \gamma_{k}$, and $\tau_{k}$ for both WOPPM and UOPPM are given by

$$
\begin{aligned}
& T_{k}=\frac{M_{k}}{\gamma_{k}} L_{k} T_{c} \\
& T_{k}=\frac{M_{k}-1+\gamma_{k}}{\gamma_{k}} L_{k} T_{c}
\end{aligned}
$$

respectively [10], [11]. In order to provide multirate and multiQoS, the code length $L_{k}$ and weight $w_{k}$ of OOCs as well as OPPM modulation parameters $\left(M_{k}\right.$ and $\left.\gamma_{k}\right)$ are designed based on the characteristics of the supported service classes.

UOPPM-OCDMA transmitter implementation is rather simple as it only involves time delaying of the optical pulses. The OCDMA encoder can be realized using an optical tapped delay line (TDL) structure which is composed of a splitter, delay elements, and a combiner as in Fig. 1. The UOPPM $M_{k}$-ary modulator for class $k$ is shown in Fig. 2. A laser pulse of width $T_{c}$ and repetition rate of $\gamma_{k} /\left(M_{k}-1+\gamma_{k}\right) \tau_{k}$ is time delayed depending on the incoming data. The serial input data is parallelized using an electronic logic unit and used to control the switches. The relative optical delays between the switches are either 0 or $2^{i-1} D_{k}$ depending on the value of input data bit $a_{i}$, where $D_{k}=\tau_{k} / \gamma_{k}$ and $i \in\left\{1,2, \ldots, \log _{2} M_{k}\right\}$. For $M_{k}$ symbols, the number of required switches is $\log _{2} M_{k}$.

At the receiver side, the OCDMA correlation decoder is composed of TDLs matched to the designed codeword. Another type of OCDMA decoder is the correlation receiver with double optical hard-limiters, Fig. 1[11]. The OPPM demodulator is merely a comparator that determines which slot in the $M_{k}$ time slots has the greatest photon count to be declared as the transmitted symbol. The all-optical PPM-demodulator proposed in [26] can be used for UOPPM systems by modifying the delays of the control signals used by an ultrafast sampling gates such that each gate samples the UOPPM frame in one of the $M_{k}$ possible OPPM slots. Shalaby in [27] has proposed an all-optical chiplevel detector for OPPM-OCDMA systems. The OPPM receiver does not require knowledge of the signal or noise power. The complexity of UOPPM-OCDMA implementation arises only for large values of $M_{k}$ [27]. A WOPPM is more complex to implement than UOPPM and OOK, both at the transmitter and receiver side. From [10], WOPPM-OCDMA signals need special techniques to be formed. For WOPPM system with $M_{k}$ and $\gamma_{k}$, each user require $\gamma_{k}$ encoder-decoder pairs, one for the first $M_{k}$ symbols and one for each $\gamma_{k}-1$ last symbols. Hence the system complexity grows with the increase of $M_{k}$ and $\gamma_{k}$.

\section{THEORETICAL ANALYSIS}

In the following sections, we will first derive expressions for the external, self, and inter interference probabilities for both multirate multi-QoS WOPPM- and UOPPM-OCDMA systems. Then, we analyze the performance of the proposed systems based on their error probabilities as functions of the number of different classes users. 


\section{A. Interference Probability}

In this section, the probabilities of external, self, and inter interferences are derived for both WOPPM- and UOPPMOCDMA systems. In our analysis, we assume that the transmissions among users are chip-synchronous which gives an upper bound on the system error probability [8].

1) Interference Probability of WOPPM-OCDMA: The probabilities of external interference from a user in class $k\left(p_{X_{k}}\right)$ and self interference (due to slots overlap) $p_{I_{k}}$ for multirate multiQoS WOPPM-OCDMA system for class $k$ can be obtained by modifying the probabilities of external and self interferences given in [24] as follows:

$$
\begin{aligned}
p_{X_{k}} & =\frac{w_{k}^{2}}{M_{k} L_{k}} \gamma_{k} \\
p_{I_{k}} & =\frac{w_{k}^{2}}{M_{k} L_{k}}\left(\gamma_{k}-1\right) .
\end{aligned}
$$

It is worth noting that for OOK-OCDMA, $M_{k}=2$ and $\gamma_{k}=1$.

To calculate the probability of inter interference from class $k$ into a class $q$ user, $p_{X_{q k}}, q \neq k$, we refer to Fig. 3. The amount of inter interference from a high-rate service class $k$ user into a low-rate service class $q$ user is different from that of inter interference from a low-rate service class $q$ user into a high-rate service class $k$ user, i.e., $p_{X_{q k}} \neq p_{X_{k q}}$. Let $j T_{c}$ be the delay of the interfering user with respect to the desired user. Since we are considering chip-synchronous case, $j$ can take values in the discrete set $\left\{0,1, \ldots, M_{k} L_{k} / \gamma_{k}-1\right\}$ with probability $\gamma_{k} / M_{k} L_{k}$. According to Fig. 3(a), for $m \in\left\{0,1, \ldots, L_{q}\right\}$ there are $m$ chips in common with the previous frame and $L_{q}-m$ chips in common with the current frame. The overlap between the previous interference frame and the desired user frame in terms of $T_{c}$ is given by:

$$
\begin{cases}m ; & \text { if } \quad 0 \leq m \leq L_{k}-1 \\ L_{k} ; & \text { if } \quad L_{k} \leq m \leq L_{q}\end{cases}
$$

while the overlap due to current interference frame is given by

$$
\begin{cases}L_{k}, & \text { if } \quad 0 \leq m \leq L_{q}-L_{k}-1 \\ -m+L_{q}, & \text { if } \quad L_{q}-L_{k} \leq m \leq L_{q} .\end{cases}
$$

Hence, the average value of $p_{X_{q k}}$ is obtained as

$$
\begin{aligned}
p_{X_{q k}}= & \frac{\gamma_{k}}{M_{k} L_{k}} \frac{w_{k} w_{q}}{L_{k}} \frac{1}{L_{q}+1}\left\{\sum_{m=0}^{L_{k}-1} m+\sum_{m=L_{k}}^{L_{q}} L_{k}\right. \\
& \left.+\sum_{m=0}^{L_{q}-L_{k}-1} L_{k}+\sum_{m=L_{q}-L_{k}}^{L_{q}}\left(-m+L_{q}\right)\right\} \\
= & \frac{\gamma_{k}}{M_{k} L_{k}} \frac{w_{k} w_{q}}{L_{k}} \frac{\left(L_{k}+2 L_{q} L_{k}-L_{k}^{2}\right)}{L_{q}+1} .
\end{aligned}
$$

For $p_{X_{k q}}, m$ can take values in the set $\left\{0,1, \ldots, L_{k}\right\}$. From Fig. 3(b), the overlap between the previous interference frame and the desired user frame in terms of $T_{c}$ is $m, m \in\left\{0,1, \ldots, L_{k}\right\}$, while the overlap between the current interference frame and the desired user frame is $-m+L_{k}, m \in$ $\left\{0,1, \ldots, L_{k}\right\}$. Hence, the average value of $p_{X_{k q}}$ is obtained as

$$
\begin{aligned}
p_{X_{k q}} & =\frac{\gamma_{q}}{M_{q} L_{q}} \frac{w_{q} w_{k}}{L_{q}} \frac{1}{L_{k}+1} \sum_{m=0}^{L_{k}} L_{k} \\
& =\frac{\gamma_{q}}{M_{q} L_{q}} \frac{w_{q} w_{k}}{L_{q}} L_{k} .
\end{aligned}
$$

2) Interference Probability of UOPPM-OCDMA: In UOPPM-OCDMA system, slot 0 has self interference just on its next slots, and slot $M_{k}-1$ has self interference just on its previous slots. However, slot $\left\lfloor\left(M_{k}+1\right) / 2\right\rfloor$ and other slots overlap with their previous and next slots. Therefore, self interference is more probable to happen when symbol $\left\lfloor\left(M_{k}+1\right) / 2\right\rfloor$ is transmitted. In our analysis, we focus on the worst case. For the case $M_{k}-1+\gamma_{k}<2 \gamma_{k}+1$, the active slot $\left\lfloor\left(M_{k}+1\right) / 2\right\rfloor$ of the desired user overlaps by $\left(1-\frac{j}{\gamma_{k}}\right) \tau_{k}, j \in\left\{1,2, \ldots, \gamma_{k}-1\right\}$, and $\left(1-\frac{1}{\gamma_{k}}\right) \tau_{k}$, with previous and next undesired slots, respectively. Hence, $p_{I_{k}}$ can be calculated as

$$
p_{I_{k}}=\sum_{\substack{i=-1 \\ i \neq 0}}^{\gamma_{k}-1} \frac{w_{k}^{2}}{M_{k} L_{k}}\left(1-\frac{|i|}{\gamma_{k}}\right)=\frac{w_{k}^{2}}{M_{k} L_{k}} \frac{\left(\gamma_{k}^{2}+\gamma_{k}-2\right)}{2 \gamma_{k}} .
$$

On the other hand, for the case $M_{k}-1+\gamma_{k} \geq 2 \gamma_{k}+1$, the active slot $\left\lfloor\left(M_{k}+1\right) / 2\right\rfloor$ of the desired user overlaps by $\left(1-\frac{|j|}{\gamma_{k}}\right) \tau_{k},|j| \in\left\{1,2, \ldots, \gamma_{k}-1\right\}$, with neighbor previous and next slots, respectively. Hence, $p_{I_{k}}$ can be calculated as

$$
p_{I_{k}}=\sum_{\substack{i=-\gamma_{k}-1 \\ i \neq 0}}^{\gamma_{k}-1} \frac{w_{k}^{2}}{M_{k} L_{k}}\left(1-\frac{|i|}{\gamma_{k}}\right)=\frac{w_{k}^{2}}{M_{k} L_{k}}\left(\gamma_{k}-1\right) .
$$

The probability of an external interference in an UOPPM technique is given by [11]

$$
p_{X_{k}}=\frac{w_{k}^{2}}{\left(M_{k}-1+\gamma_{k}\right) L_{k}} \gamma_{k} .
$$

Using a similar procedure to that used in WOPPM-OCDMA with the difference that $j$ can take values in the discrete set $\left\{0,1, \ldots,\left(M_{k}-1+\gamma_{k}\right) L_{k} / \gamma_{k}-1\right\}$ with probability $\gamma_{k} /\left(M_{k}-1+\gamma_{k}\right) L_{k}$, we can find that $p_{X_{q k}}$ and $p_{X_{k q}}$ are given by

$$
\begin{aligned}
& p_{X_{q k}}=\frac{\gamma_{k}}{\left(M_{k}-1+\gamma_{k}\right) L_{k}} \frac{w_{k} w_{q}}{L_{k}} \frac{\left(L_{k}+2 L_{q} L_{k}-L_{k}^{2}\right)}{L_{q}+1} \\
& p_{X_{k q}}=\frac{\gamma_{q}}{\left(M_{q}-1+\gamma_{q}\right) L_{q}} \frac{w_{q} w_{k}}{L_{q}} L_{k}
\end{aligned}
$$

respectively.

\section{B. Bit Error Probabilities}

1) BER of a Correlation Receiver: In a correlation receiver of a class $k$ user, an error occurs when the number of received pulses $Z_{j}, j \in \mathcal{M}_{k}$ from all weighted chips of at least one undesired slot exceeds a threshold $T h=w_{k}$. The probability of 
symbol error of class $k$ user $P_{S}(k)$ is then

$$
P_{S}(k)=\sum_{j=0}^{M_{k}-1} P[E \mid j] \operatorname{Pr}\{D=j\}
$$

where $\mathrm{D}$ is a random variable that denotes the transmitted data symbol of class $k$. Under the assumption that the transmitted data is equally likely, i.e., $\operatorname{Pr}\{D=j\}=1 / M_{k}$, then $P[E \mid j]$ is independent of $j$. Without loss of generality, suppose that the symbol 0 is transmitted, i.e., $D=0$. Employing a union bound, the symbol error probability can be given as

$$
\begin{aligned}
P_{S}(k) & =P[E \mid 0] \\
& =\operatorname{Pr}\left\{Z_{j} \geq w_{k}, \text { some } j \neq 0 \mid D=0\right\} \\
& \leq \sum_{j=1}^{M_{k}-1} \operatorname{Pr}\left\{Z_{j} \geq w_{k} \mid D=0\right\} \\
& =\left(M_{k}-1\right) \operatorname{Pr}\left\{Z_{1} \geq w_{k} \mid D=0\right\} .
\end{aligned}
$$

The last probability can be obtained as

$$
\begin{aligned}
& \operatorname{Pr}\left\{Z_{1} \geq w_{k} \mid D=0\right\} \\
& =\sum_{\ell_{1}=0}^{N_{1}} \cdots \sum_{\ell_{k}=0}^{N_{k}-1} \cdots \sum_{\ell_{Q}=0}^{N_{Q}}\left(\begin{array}{c}
N_{k}-1 \\
\ell_{k}
\end{array}\right) p_{X_{k}}^{\ell_{k}}\left(1-p_{X_{k}}\right)^{N_{k}-1-\ell_{k}} \\
& \quad \times \prod_{\substack{q=1 \\
q \neq k}}^{Q}\left(\begin{array}{c}
N_{q} \\
\ell_{q}
\end{array}\right) p_{X_{k q}}^{\ell_{q}}\left(1-p_{X_{k q}}\right)^{N_{q}-\ell_{q}}\left[\Omega\left(\sum_{i=1}^{Q} \ell_{i}-w_{k}\right)\right. \\
& \left.\quad+p_{I_{k}}\left\{\Omega\left(\sum_{i=1}^{Q} \ell_{i}-\left(w_{k}-1\right)\right)-\Omega\left(\sum_{i=1}^{Q} \ell_{i}-w_{k}\right)\right\}\right]
\end{aligned}
$$

where the indicator function

$$
\Omega(x) \stackrel{\text { def }}{=} \begin{cases}1, & \text { if } \quad x \geq 0 \\ 0, & \text { otherwise. }\end{cases}
$$

2) BER of a Correlation Receiver With a Hard-Limiter: For the correlation receiver with hard-limiter of a class $k$ user, an error occurs only when the number of interfering pulses in every weighted mark position of at least one undesired slot is nonzero. Given that there are $\ell$ interfering users, each interferes at exactly one mark position, there is a set of possible interference patterns. To describe these patterns, we define a $Q \times w_{k}$ interference matrix $\mathscr{L}$ whose element $\ell_{i j}^{k}, i \in \chi_{k}=\left\{1,2, \ldots, w_{k}\right\}, j \in \mathcal{M}_{k}, k \in \Omega$, represents the number of users (out of available $\ell_{j}^{k}$ class $k$ users) that interfere with chip number $i$ of the mark positions of slot $j$,

$$
\mathscr{L} \stackrel{\text { def }}{=}\left(\begin{array}{cccc}
\ell_{1 j}^{1} & \ell_{2 j}^{1} & \ldots & \ell_{w_{k} j}^{1} \\
\ell_{1 j}^{2} & \ell_{2 j}^{2} & \ldots & \ell_{w_{k} j}^{2} \\
\ldots & & \ldots & \\
\ell_{1 j}^{Q} & \ell_{2 j}^{Q} & \ldots & \ell_{w_{k} j}^{Q}
\end{array}\right) \text {. }
$$

Notice that the sum over the rows of $\mathscr{L}$ gives the vector $\ell$, defined as

$$
\boldsymbol{\ell}=\left\{\ell_{j}^{1}, \ell_{j}^{2}, \ldots, \ell_{j}^{Q}\right\} \stackrel{\text { def }}{=}\left\{\sum_{i=1}^{w_{k}} \ell_{i j}^{1}, \sum_{i=1}^{w_{k}} \ell_{i j}^{2}, \ldots, \sum_{i=1}^{w_{k}} \ell_{i j}^{Q}\right\} .
$$

On the other hand, the sum over the columns of $\mathscr{L}$ gives the vector $\boldsymbol{\alpha}$, defined as

$$
\begin{aligned}
& \boldsymbol{\alpha}=\left\{\alpha_{1 j}, \alpha_{2 j}, \ldots, \alpha_{w_{k} j}\right\} \\
& \stackrel{\text { def }}{=}\left\{\sum_{k=1}^{Q} \ell_{1 j}^{k}, \sum_{k=1}^{Q} \ell_{2 j}^{k}, \ldots, \sum_{k=1}^{Q} \ell_{w_{k} j}^{k}\right\}
\end{aligned}
$$

Here $\alpha_{i j}, i \in \chi_{k}, j \in \mathcal{M}_{k}$, represents the number of interfering users that overlap with class $k$ chip number $i$ of the mark positions of slot $j$. The symbol error probability $P_{S}(k)$ of class $k$ user with $w_{k}$ code weight given $\mathcal{N}$ users can be evaluated as

$$
\begin{aligned}
P_{S}(k)= & \sum_{\ell_{j}^{1}=0}^{N_{1}} \ldots \sum_{\ell_{j}^{k}=0}^{N_{k}-1} \cdots \sum_{\ell_{j}^{Q}=0}^{N_{Q}} \operatorname{Pr}\{\boldsymbol{\ell} \text { interferers } \mid \mathcal{N} \text { users }\} \\
& \times \operatorname{Pr}_{(k)}\{\text { error } \mid \boldsymbol{\ell} \text { interferers }\} .
\end{aligned}
$$

The first conditional probability in the right-hand side of (20) can be evaluated as follows:

$$
\begin{aligned}
& \operatorname{Pr}\{\boldsymbol{\ell} \text { interferers } \mid \mathcal{N} \text { users }\} \\
& =\left(\begin{array}{c}
N_{k}-1 \\
\ell_{j}^{k}
\end{array}\right) p_{X_{k}}^{\ell_{j}^{k}}\left(1-p_{X_{k}}\right)^{N_{k}-1-\ell_{j}^{k}} \\
& \quad \times \prod_{\substack{q=1 \\
q \neq k}}^{Q}\left(\begin{array}{c}
N_{q} \\
\ell_{j}^{q}
\end{array}\right) p_{X_{k q}}^{\ell_{j}^{q}}\left(1-p_{X_{k q}}\right)^{N_{q}-\ell_{j}^{q}} .
\end{aligned}
$$

Considering that symbol 0 is transmitted and using the union bound, $\operatorname{Pr}_{(k)}\{$ error $\mid \ell$ interferers $\}$ can be calculated as

$$
\begin{aligned}
& \operatorname{Pr}_{(k)}\{\text { error } \mid \boldsymbol{\ell} \text { interferers }\} \\
& =\sum_{j=0}^{M_{k}-1} \operatorname{Pr}_{(k)}\{\text { error } \mid \boldsymbol{\ell} \text { interferers, } D=j\} \operatorname{Pr}\{D=j\} \\
& =\operatorname{Pr}_{(k)}\{\text { error, some } j \neq 0 \mid \ell \text { interferers, } D=0\} \\
& \leq \sum_{j=1}^{M_{k}-1} \operatorname{Pr}_{(k)}\{\text { error } \mid \boldsymbol{\ell} \text { interferers, } D=0\} \\
& =\left(M_{k}-1\right) \operatorname{Pr}_{(k)}\{\text { error } \mid \ell \text { interferers, } D=0\} .
\end{aligned}
$$

Let the random variable $T_{(k)}$ denote the number of interfered mark positions of an undesired slot belonging to the desired class $k$ user. The last probability can be evaluated as [28]

$$
\begin{aligned}
& \operatorname{Pr}_{(k)}\{\text { error } \mid \ell \text { interferers, } D=0\} \\
& =\operatorname{Pr}\left\{T_{(k)}=w_{k} \mid \ell \text { interferers }\right\} \\
& \quad+\operatorname{Pr}\left\{T_{(k)}=w_{k}-1, \nu_{I_{k}}=1 \mid \ell \text { interferers }\right\}
\end{aligned}
$$


where $\nu_{I_{k}} \in\{0,1\}$ is the number of pulses that cause self interference from slot 0 onto other undesired slots. The average probability of self interference on a specific mark of an undesired slot in class $k$ is $\frac{1}{w_{k}} p_{I_{k}}$. Since $\left\{T_{(k)}=w_{k}-1 \mid \ell\right.$ interferers $\}$ and $\left\{\nu_{I_{k}}=1 \mid \ell\right.$ interferers $\}$ are independent, we can write [28]

$$
\begin{aligned}
& \operatorname{Pr}\left\{T_{(k)}=w_{k}-1, \nu_{I_{k}}=1 \mid \ell \text { interferers }\right\} \\
& =\operatorname{Pr}\left\{\nu_{I_{k}}=1 \mid \ell \text { interferers }\right\} \\
& \quad \times \operatorname{Pr}\left\{T_{(k)}=w_{k}-1 \mid \ell \text { interferers }\right\} \\
& =\frac{1}{w_{k}} p_{I_{k}} \times \operatorname{Pr}\left\{T_{(k)}=w_{k}-1 \mid \ell \text { interferers }\right\}
\end{aligned}
$$

Employing a similar technique to that used in [29], we get

$$
\begin{aligned}
\operatorname{Pr} & \left\{T_{(k)}=w_{k} \mid \ell \text { interferers }\right\} \\
& =\operatorname{Pr}\left\{\alpha_{1 j} \geq 1, \alpha_{2 j} \geq 1, \ldots, \alpha_{w_{k} j} \geq 1 \mid \ell \text { interferers }\right\} \\
& =\frac{1}{w_{k}^{\ell}} S\left(w_{k}, \ell\right)
\end{aligned}
$$

where $S\left(w_{k}, \ell\right)$ represents the number of patterns in which all $w_{k}$ marked chips of one of the undesired slots of class $k$ user (i.e., $j \neq 0$ ) are hit at least by one interfering pulse and can be computed as shown below

$$
\begin{aligned}
S\left(w_{k}, \ell\right)= & w_{k}^{\ell_{j}^{1}+\cdots+\ell_{j}^{Q}}-\left(\begin{array}{c}
w_{k} \\
1
\end{array}\right)\left(w_{k}-1\right)^{\ell_{j}^{1}+\cdots+\ell_{j}^{Q}} \\
& +\left(\begin{array}{c}
w_{k} \\
2
\end{array}\right)\left(w_{k}-2\right)^{\ell_{j}^{1}+\cdots+\ell_{j}^{Q}}+\cdots+(-1)^{w_{k}-1} \\
& \times\left(\begin{array}{c}
w_{k} \\
w_{k}-1
\end{array}\right)\left(w_{k}-\left(w_{k}-1\right)\right)^{\ell_{j}^{1}+\cdots+\ell_{j}^{Q}} \\
& =\sum_{t=0}^{w_{k}-1}(-1)^{t}\left(\begin{array}{c}
w_{k} \\
t
\end{array}\right)\left(w_{k}-t\right)^{\ell} .
\end{aligned}
$$

The last probability in (24) can be evaluated as follows [28]:

$$
\begin{aligned}
& \operatorname{Pr}\left\{T_{(k)}=w_{k}-1 \mid \ell \text { interferers }\right\}=w_{k} \\
& \quad \times \operatorname{Pr}\left\{\alpha_{1 j} \geq 1, \ldots, \alpha_{w_{k}-1 j} \geq 1, \alpha_{w_{k} j}=0 \mid \ell \text { interferers }\right\} \\
& =w_{k} \times\left(\operatorname{Pr}\left\{\alpha_{1 j} \geq 1, \ldots, \alpha_{w_{k}-1 j} \geq 1 \mid \ell \text { interferers }\right\}\right. \\
& \left.\quad-\operatorname{Pr}\left\{\alpha_{1 j} \geq 1, \ldots, \alpha_{w_{k}-1 j} \geq 1, \alpha_{w_{k} j} \geq 1 \mid \ell \text { interferers }\right\}\right)
\end{aligned}
$$

where $\operatorname{Pr}\left\{\alpha_{1 j} \geq 1, \ldots, \alpha_{w_{k}-1 j} \geq 1 \mid \ell\right.$ interferers $\}$ is given by

$$
\begin{aligned}
\operatorname{Pr} & \left\{\alpha_{1 j} \geq 1, \ldots, \alpha_{w_{k}-1 j} \geq 1 \mid \ell \text { interferers }\right\} \\
& =\frac{1}{\left(w_{k}-1\right)^{\ell}} S\left(w_{k}-1, \ell\right) \\
& =\frac{1}{\left(w_{k}-1\right)^{\ell}} \sum_{t=0}^{w_{k}-2}(-1)^{t}\left(\begin{array}{c}
w_{k}-1 \\
t
\end{array}\right)\left(w_{k}-1-t\right)^{\ell} .
\end{aligned}
$$

Substituting in (20), we obtain an upper bound on the probability of symbol error of a class $k$ user $P_{S}(k)$ for a correlation receiver with a hard-limiter as

$$
\begin{aligned}
& P_{S}(k) \leq\left(M_{k}-1\right) \sum_{\ell_{j}^{1}=0}^{N_{1}} \ldots \sum_{\ell_{j}^{k}=0}^{N_{k}-1} \cdots \sum_{\ell_{j}^{Q}=0}^{N_{Q}} \\
& \times\left(\begin{array}{c}
N_{k}-1 \\
\ell_{j}^{k}
\end{array}\right) p_{X_{k}}^{\ell_{j}^{k}}\left(1-p_{X_{k}}\right)^{N_{k}-1-\ell_{j}^{k}} \\
& \times \prod_{\substack{q=1 \\
q \neq k}}^{Q}\left(\begin{array}{c}
N_{q} \\
\ell_{j}^{q}
\end{array}\right) p_{X_{k q}}^{\ell_{j}^{q}}\left(1-p_{X_{k q}}\right)^{N_{q}-\ell_{j}^{q}} \\
& \times\left\{\frac{1}{w_{k}^{\ell}} \sum_{t=0}^{w_{k}-1}(-1)^{t}\left(\begin{array}{c}
w_{k} \\
t
\end{array}\right)\left(w_{k}-t\right)^{\ell}+p_{I_{k}}\right. \\
& \times\left[\frac{1}{\left(w_{k}-1\right)^{\ell}} \sum_{t=0}^{w_{k}-2}(-1)^{t}\left(\begin{array}{c}
w_{k}-1 \\
t
\end{array}\right)\left(w_{k}-1-t\right)^{\ell}\right. \\
& \left.\left.-\frac{1}{w_{k}^{\ell}} \sum_{t=0}^{w_{k}-1}(-1)^{t}\left(\begin{array}{c}
w_{k} \\
t
\end{array}\right)\left(w_{k}-t\right)^{\ell}\right]\right\} \text {. }
\end{aligned}
$$

Notice that the above equation is also valid for OOK-OCDMA by replacing $\left(M_{k}-1\right)$ with $1 / 2$. Finally, the BER $P_{b}(k)$ can be obtained using the probability of symbol error from the famous relation [30]

$$
P_{b}(k)=\frac{M_{k}}{2\left(M_{k}-1\right)} P_{S}(k) .
$$

\section{NUMERICAL RESULTS}

In our numerical results, we consider a three-class OCDMA network defined as follows:

1) Class 1: low rate and low QoS, $\left(R_{01}=5.78 \times 10^{-4}\right.$ nats/chip, BER $\leq 10^{-4}$ ).

2) Class 2: medium rate and medium QoS, $\left(R_{02}=1.16 \times\right.$ $10^{-3}$ nats/chip, BER $\leq 10^{-6}$ ).

3) Class 3: high rate and high QoS, $\left(R_{03}=1.73 \times 10^{-3}\right.$ nats/chip, BER $\leq 10^{-9}$ ).

For OOK-OCDMA, the code length $L_{k}$ of class $k$ is determined using $R_{0 k}=\log 2 / L_{k}^{\mathrm{OOK}}$. Hence, $L_{1}^{\mathrm{OOK}}=$ $1200, L_{2}^{\mathrm{OOK}}=600$, and $L_{3}^{\mathrm{OOK}}=400$. The code-set parameters, $N_{k}, L_{k}$, and $w_{k}$ are related with the Johnson bound as [25]

$$
N_{k} \leq \frac{L_{k}-1}{w_{k}\left(w_{k}-1\right)} .
$$

The number of available codewords in class 1 , class 2 , and class 3 is set to 28,15 and 7, respectively. The code weight of class 2 and class 3 is selected as the maximum value that satisfies the Johnson bound. Hence, $w_{2}=6$ and $w_{3}=8$. While the code weight of class 1 (low QoS) is chosen so as to satisfy both the condition in (2) and Johnson bound, i.e., $w_{1}=4$. To carry out a fair comparison between different modulation schemes (OOK, WOPPM, and UOPPM), the values of chip duration $T_{c}$, the throughput $R_{0 k}$ of each class $k$ user, and the number of available codwords are kept constant among all service classes. 


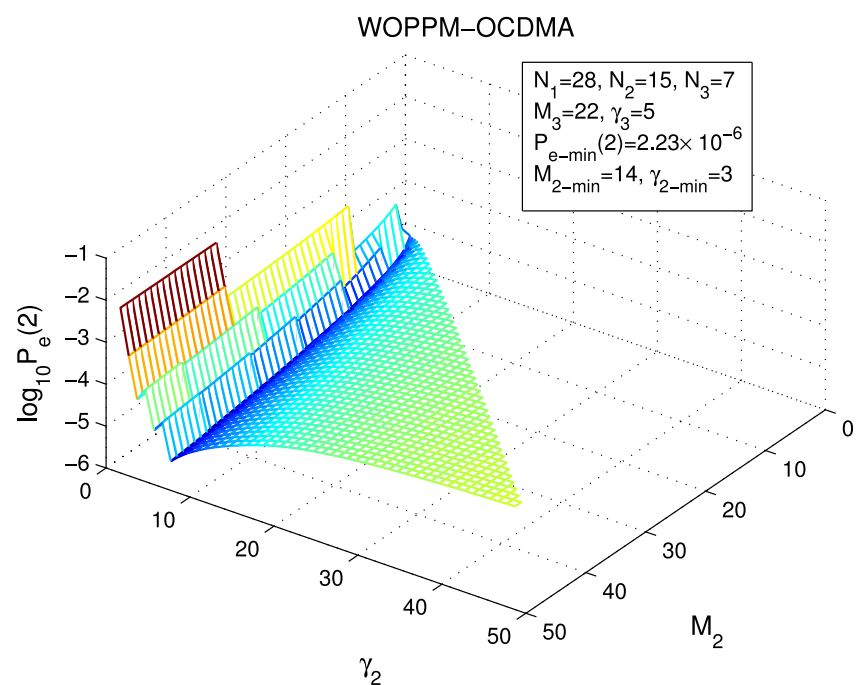

(a)

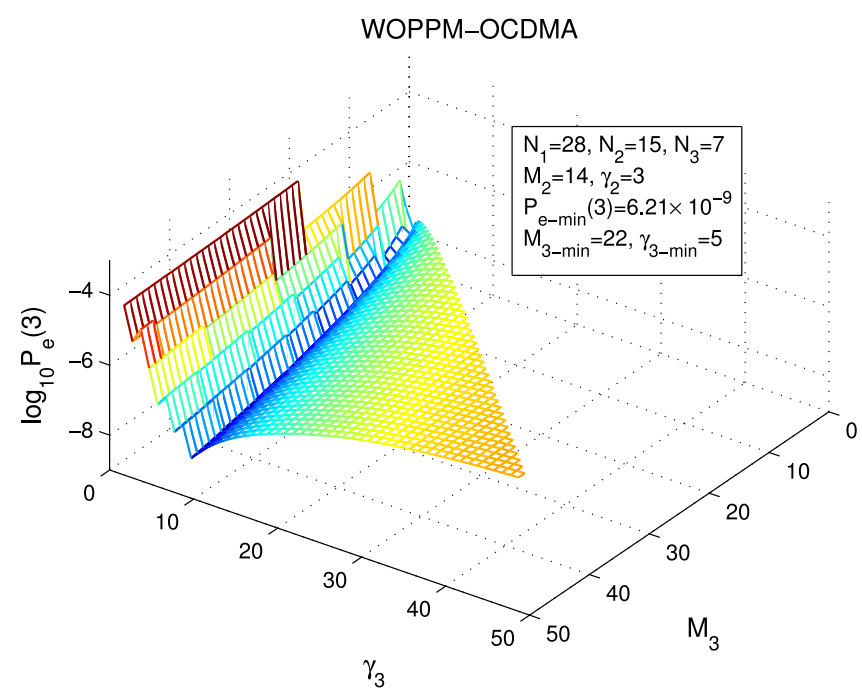

(b)

Fig. 4. Bit-error probability for WOPPM-OCDMA systems for (a) class 2 users versus $M_{2}$ and $\gamma_{2}$, and (b) class 3 users versus $M_{3}$ and $\gamma_{3}$.

\section{A. Unconstrained Frame Length}

In this case, there is no constraint on the frame length. Proper choice of parameters can improve network performance, i.e., minimize the BER and maximize the capacity. So it is important to find an optimum value for each parameter to get the best performance from the network. According to the network usage, some parameters such as the number of users $N_{k}$ and throughput $R_{0 k}$ of class $k \in \Omega$ are known. Other parameters, such as $M_{k}$ and $\gamma_{k}$, should be found properly. To simplify the parameters selection process, we assume that low-rate service-class (i.e., class 1) users send their data using OOK-OCDMA scheme, while other classes users send their data using W/UOPPM-OCDMA scheme. The code lengths for classes adopting WOPPM- and UOPPM-OCDMA signaling are given by

$$
\begin{gathered}
L_{k}^{\mathrm{WOPPM}}=\frac{\gamma_{k} \log _{2} M_{k} L_{k}^{\mathrm{OOK}}}{M_{k}}, k \neq 1 \\
L_{k}^{\mathrm{UOPPM}}=\frac{\gamma_{k} \log _{2} M_{k} L_{k}^{\mathrm{OOK}}}{M_{k}-1+\gamma_{k}}, k \neq 1
\end{gathered}
$$

respectively, and the code weights are selected as the maximum values that satisfy both the Johnson bound and the condition in (2). Proper choice of both $M_{k}$ and $\gamma_{k}$ values to minimize BER is not straightforward. In order to have some insight on the system design, 3-D graphs showing the variations of upper bound probabilities of bit error of both WOPPM- and UOPPM-OCDMA systems (in the presence of hard-limiters) versus $M_{k}$ and $\gamma_{k}$ are plotted in Figs. 4 and 5, respectively, with $N_{1}=28, N_{2}=15, N_{3}=7, R_{01}=5.78 \times$ $10^{-4}, R_{02}=1.16 \times 10^{-3}$, and $R_{03}=1.73 \times 10^{-3}$. It is worth noting that $\gamma_{k}$ can takes values only in the range $\left\{1,2, \ldots, M_{k}-\right.$ $1\}$. At small values of $\gamma_{k}$, from (32) $L_{k}^{W / \text { UOPPM }}$ is smaller than $L_{k}^{\mathrm{OOK}}$ for the same throughput. Hence, to support the same number of codewords available in OOK system, according to Johnson bound we should use lower code weights which results in higher BER. As $\gamma_{k}$ increases, we can use codewords with larger weights, and hence the BER decreases. However at higher $\gamma_{k}$, the probability of bit error starts to increase due to the increase in probabilities of external and self interferences. In Fig. 4(a), it is assumed that $M_{3}=22$ and $\gamma_{3}=5$, while in Fig. 4(b), $M_{2}=14$ and $\gamma_{2}=3$. According to Fig. 4, the minimum probabilities of bit errors for WOPPM-OCDMA systems are approximately $2.23 \times 10^{-6}$ and $6.21 \times 10^{-9}$ for classes 2 and 3 , respectively, and are achieved when $\left(M_{2}, \gamma_{2}\right)=(14,3)$ and $\left(M_{3}, \gamma_{3}\right)=(22,5)$, respectively. From Fig. 5, the minimum probabilities of bit errors for UOPPM-OCDMA systems are approximately $2.46 \times 10^{-6}$ and $5.49 \times 10^{-9}$ for classes 2 and 3 , respectively, and are achieved when $\left(M_{2}, \gamma_{2}\right)=(11,3)$ and $\left(M_{3}, \gamma_{3}\right)=(21,6)$, respectively.

Considering the values of $M_{2}, \gamma_{2}, M_{3}$, and $\gamma_{3}$ obtained from Figs. 4 and 5 for WOPPM- and UOPPM-OCDMA system, respectively, the BER performance of the three service-classes is plotted in Fig. 6 using correlation receiver with and without hard-limiter as a function of the number of users in class 2. For correlation receiver with hard-limiter, the number of users in class 1 and class 3 are fixed to 14 and 5, respectively. For correlation receiver without hard-limiters, however, the number of users in class 1 and class 3 are fixed to 10 and 3, respectively. Then, the total number of supported users in the network using correlation receiver with hard-limiter under the prescribed BER for the three classes are 29, 34, and 32 for OOK-, WOPPM-, and UOPPMOCDMA systems, respectively. From Fig. 6(a), in order to meet the BER requirements of the three service-classes, $N_{2}$ cannot exceed eight for OOK, but it can be as large as 15 and 13 for WOPPM and UOPPM, respectively. On the other hand, from Fig. 6(b) for correlation receiver without hard-limiters, OOK can support up to 4 class 2 users to meet BER requirements, while WOPPM and UOPPM can support up to 8 and 7 users, respectively. Therefore, using correlation receiver, the network can support 17, 21, and 20 total users under the prescribed BER for OOK-, WOPPM-, and UOPPM-OCDMA systems, respectively. From these results, only WOPPM- and UOPPM-OCDMA 
UOPPM-OCDMA

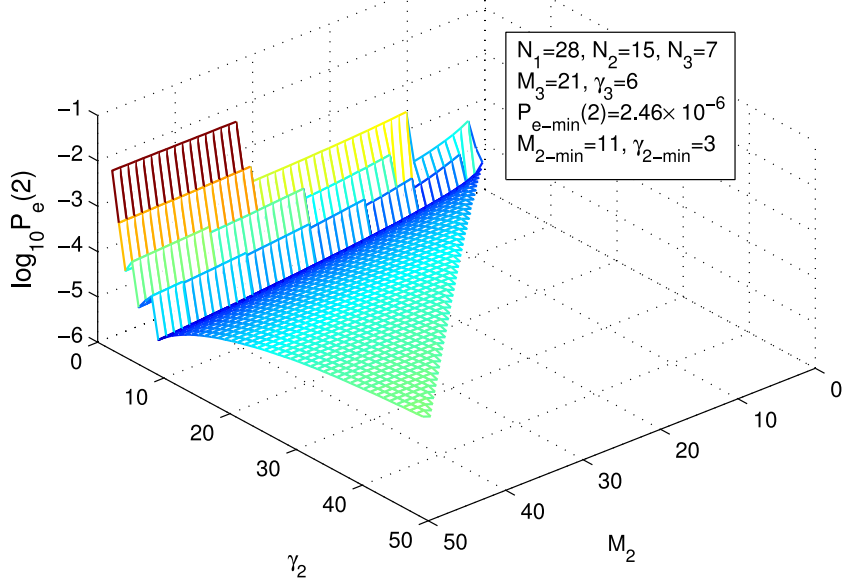

(a)
UOPPM-OCDMA

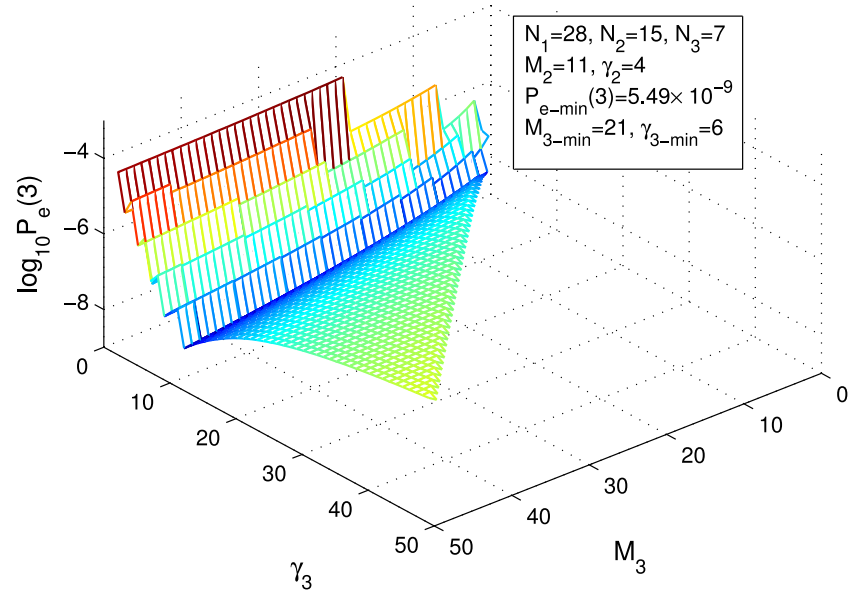

(b)

Fig. 5. Bit-error probability for UOPPM-OCDMA systems for (a) class 2 users versus $M_{2}$ and $\gamma_{2}$, and (b) class 3 users versus $M_{3}$ and $\gamma_{3}$.

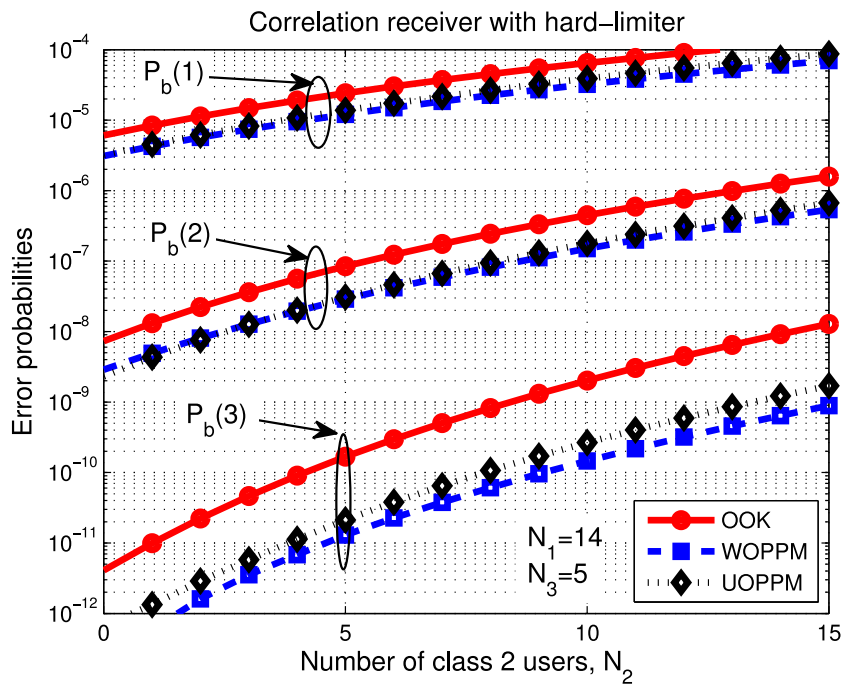

(a)

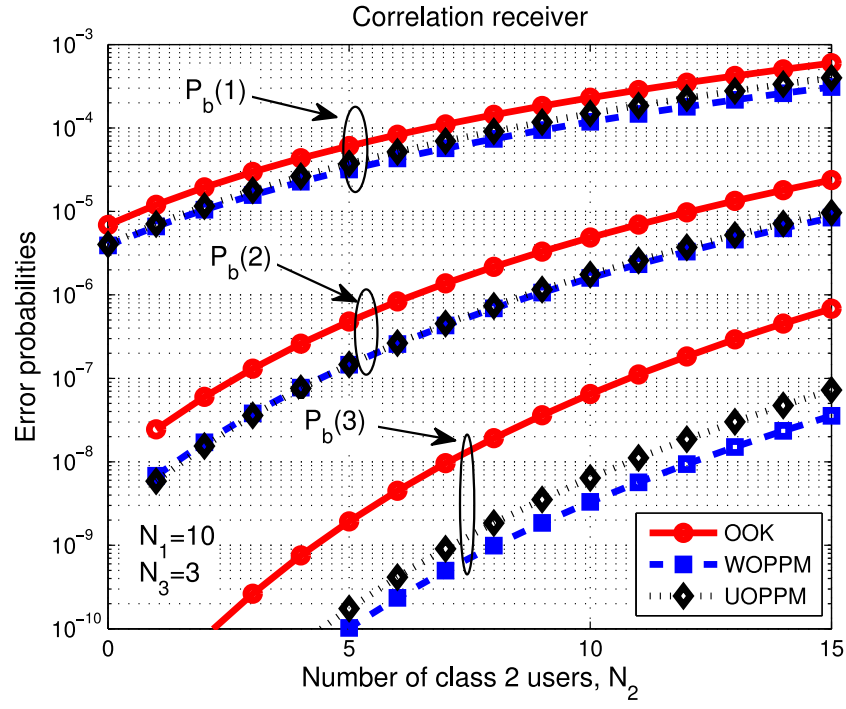

(b)

Fig. 6. BER for three-class OOK-, WOPPM-, and UOPPM-OCDMA systems versus number of active users in class 2. (a) Correlation receiver with hard-limiter. (b) Correlation receiver without hard-limiters.

systems using correlation receivers with hard-limiters are suited to a typical fiber-to-the-premise (FTTP) network, which typically has 32 users. The proposed WOPPM- and UOPPMOCDMA systems can provide data rates above $2.25 \mathrm{~Gb} / \mathrm{s}\left(T_{c}=\right.$ $0.02 \mathrm{~ns}$ ) shared among 32 users with different data rate and QoS requirements.

\section{B. Constrained Frame Length}

In this section, in order to simplify the parameters selection process for a W/UOPPM-OCDMA system, we keep the frame length $T_{k}$ fixed for all classes. For three-class system, it is as- sumed that the parameters $N_{k}, R_{0 k}, M_{1}$ and $\gamma_{1}$ (and hence $L_{1}$ ) are chosen by the network designer and the values of $\left(M_{2}, \gamma_{2}\right)$ and $\left(M_{3}, \gamma_{3}\right)$ are to be found such that the network supports a number of users $\Gamma_{k}^{T h}$ having a service with a specific maximum acceptable BER, $\mathrm{BER}_{k}^{\max }$ in class $k$. The procedure of selecting the parameter values is summarized as

1) Calculate $M_{k}, k \in\{2,3\}$ using

$$
M_{k}= \begin{cases}\left\lceil e^{M_{1} L_{1} R_{0 k} / \gamma_{1}}\right\rceil ; & \text { for WOPPM-OCDMA } \\ \left\lceil e^{\left(M_{1}-1+\gamma_{1}\right) L_{1} R_{0 k} / \gamma_{1}}\right\rceil ; & \text { for UOPPM-OCDMA. }\end{cases}
$$




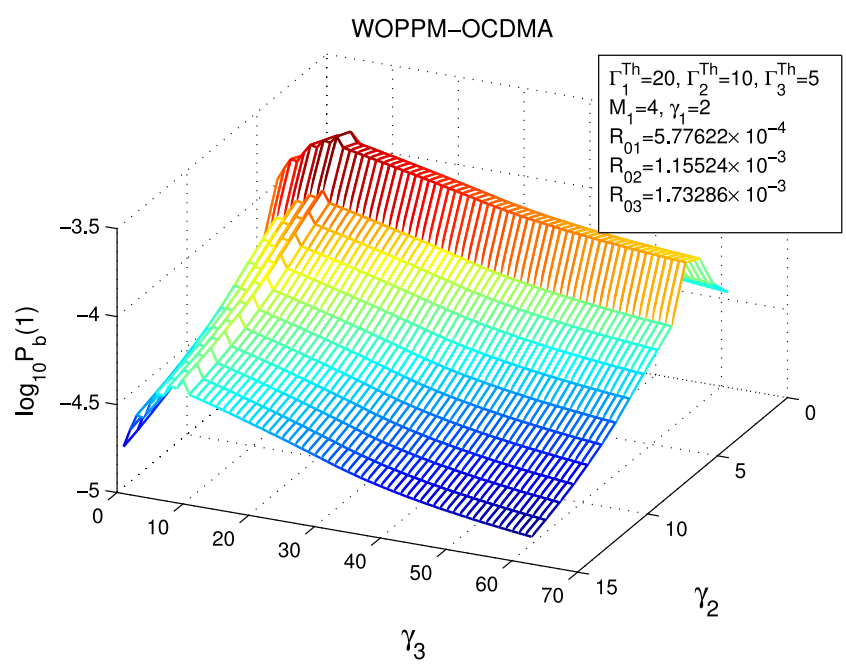

(a)

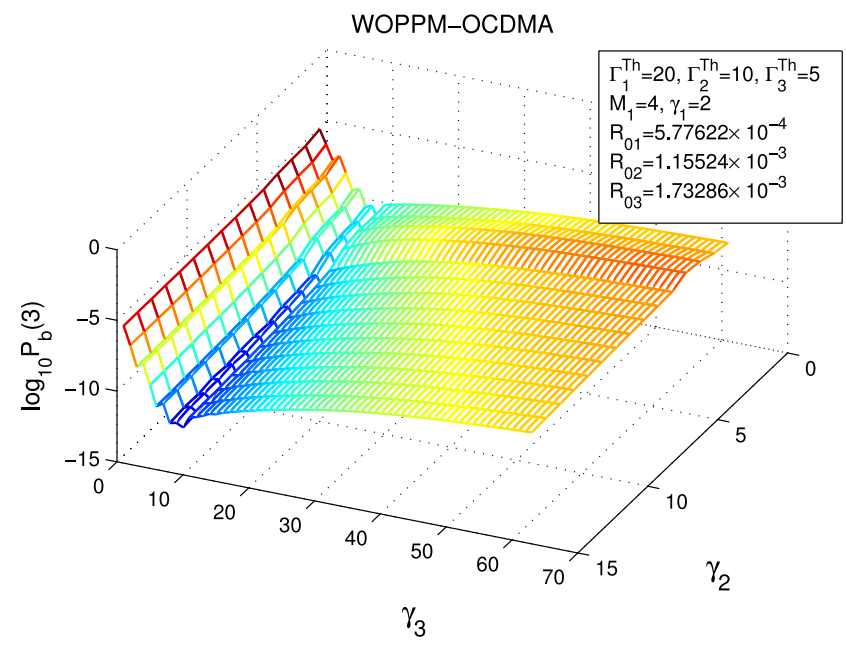

(c)

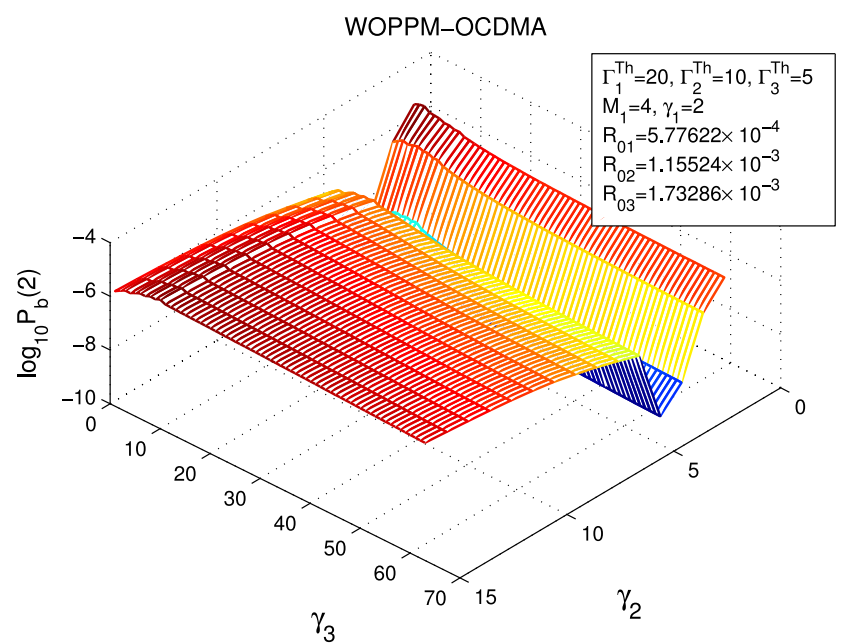

(b)

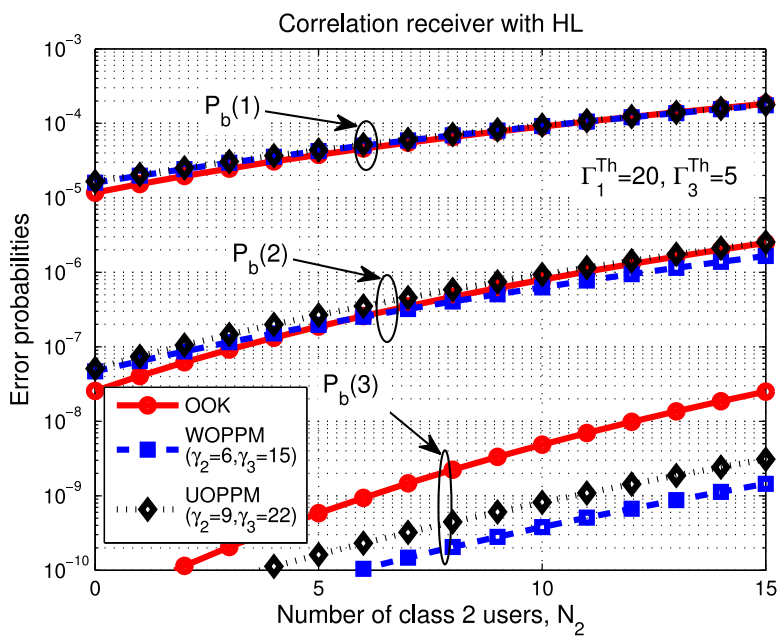

(d)

Fig. 7. (a) BER for Class 1 receivers versus $\gamma_{2}$ and $\gamma_{3}$. (b) BER for Class 2 receivers versus $\gamma_{2}$ and $\gamma_{3}$. (c) BER for Class 3 receivers versus $\gamma_{2}$ and $\gamma_{3}$. (d) BER for three-class OOK-, WOPPM- and UOPPM-OCDMA systems versus $\mathrm{N}_{2}$.

2) Calculate $L_{k}, k \in\{2,3\}$ for all possible values of $\gamma_{k} \in$ $\left\{1,2, \ldots, M_{k}-1\right\}$ using

$$
L_{k}= \begin{cases}\frac{\gamma_{k} \log M_{k}}{M_{k} R_{0 k}} ; & \text { for WOPPM-OCDMA } \\ \frac{\gamma_{k} \log _{M_{k}}}{\left(M_{k}-1+\gamma_{k}\right) R_{0 k}} ; & \text { for UOPPM-OCDMA. }\end{cases}
$$

3) Plot BER versus $\gamma_{2}$ and $\gamma_{3}$ as 3-D graph for different service classes at $\Gamma_{k}^{T h}$.

4) In each plot there exist some points $\left(\gamma_{2}, \gamma_{3}\right)$ in which BER $\leq \mathrm{BER}_{k}^{\max }$ of class $k$.

5) The intersection of these points that achieves BER requirements for all classes represents the proper values for $\gamma_{2}$ and $\gamma_{3}$.

We have done this for our network as an example. It is required to support $\Gamma_{1}^{T h}=20$ at $\mathrm{BER}_{1}^{\max }=10^{-4}, \Gamma_{2}^{T h}=10$ at $\mathrm{BER}_{2}^{\max }=10^{-6}$, and $\Gamma_{3}^{T h}=5$ at $\mathrm{BER}_{3}^{\max }=10^{-9}$ (35 users available in the network). Both $N_{k}$ and $R_{0 k}$ are as described above. $M_{1}=4$ and $\gamma_{1}=2$ for WOPPM and $M_{1}=4$ and $\gamma_{1}=3$ for UOPPM (to get the same code length as $L_{1}^{\mathrm{OOK}}$ ). For every value of $M_{k}$ and $\gamma_{k}$, the code length is calculated from (34) for W/UOPPM-OCDMA and the maximum possible value for the code weight are obtained using (2) and (31). The resulting BERs for class 1 , class 2 , and class 3 are plotted as a 3-D graph in Fig. 7(a)- (c) for WOPPM-OCDMA system using correlation receiver with hard-limiter. The intersection points $\left(\gamma_{2}, \gamma_{3}\right)$ for WOPPM-OCDMA system are: $(6,6),(6,7),(6,12)$, $(6,13),(6,14),(6,15),(6,16),(6,17),(7,15),(7,16),(7,17),(7,18)$, and $(7,19)$. The corresponding points for UOPPM-OCDMA system (not shown in Figure) are: $(8,22),(9,20),(9,21),(9,22)$, and $(9,23)$. The bit error probabilities for different signaling schemes (OOK, WOPPM, and UOPPM) using correlation receiver with hard-limiter are shown in Fig. 7(d) for $\Gamma_{1}^{T h}=20, \Gamma_{3}^{T h}=5$ versus the number of users in class 2 . According to the figure, $\mathrm{OOK}$ system cannot support (only support 6 users from class 2 ) the required users with required BERs, while $N_{2}=\Gamma_{2}^{T h}=10$ are supported using either WOPPM- or UOPPM-OCDMA. 


\section{CONCLUSION}

In this paper, a multirate multi-QoS transmission system using OPPM techniques, coupled with MLVW-OCDMA as a signature code, has been proposed and its performance has been analyzed. Two modulation techniques have been considered, namely, WOPPM and UOPPM. Upper bounds on the probabilities of error for the proposed system have been obtained for both correlation receivers with and without hard-limiters. Numerical results have been evaluated for OOK, WOPPM, and UOPPM signaling schemes. All systems are assumed to work under same laser pulse width, number of available codewords, and throughput in each service class. Our results show that by properly choosing both $M_{k}$ and $\gamma_{k}$ for service class $k$, W/UOPPMOCDMA systems outperform OOK-OCDMA systems in terms of BER and the maximum number of users that can be accommodated at a specific maximum acceptable BER. Moreover, we have suggested two simple system design procedures that can be used to choose the proper values of modulation parameters $\left(M_{k}, \gamma_{k}\right)$ for class $k$, specifically constrained and unconstrained frame length.

Future work will focus on obtaining some empirical solutions that can be easily used to estimate the optimum values of each parameter in W/UOPPM-OCDMA systems. It would also be interesting in the near future to validate our theoretical results using numerical simulations (e.g., RSoft optSIM).

\section{REFERENCES}

[1] K. Kitayama, X. Wang, and N. Wada, "OCDMA over WDM PON-solution path to gigabit-symmetric FTTH," J. Lightw. Technol., vol. 24, no. 4, pp. 1654-62, Apr. 2006.

[2] C.-C. Yang, "High speed and secure optical CDMA-based passive optical networks," Comput. Netw., vol. 53, no. 12, pp. 2182-2191, Aug. 2009.

[3] J. S. Vardakas, I. D. Moscholios, M. D. Logothetis, and V. G. Stylianakis, "Performance analysis of OCDMA PONs supporting multi-rate bursty traffic," Trans. Commun., vol. 61, no. 8, pp. 3374-3384, Aug. 2013.

[4] H. Beyranvand and J. A. Salehi, "Multirate and multi-quality-of-service passive optical network based on hybrid WDM/OCDM system," IEEE Commun. Mag., vol. 49, no. 2, pp. S39-S44, Feb. 2011.

[5] A. Stok and E. Sargent, "Lighting the local area: optical code-division multiple access and quality of service provisioning," IEEE Netw., vol. 14, no. 6, pp. 42-46, Nov./Dec. 2000.

[6] A. Stok and E. H. Sargent, "The role of optical CDMA in access networks," IEEE Commun. Mag., vol. 40, no. 9, pp. 83-87, Sep. 2002.

[7] J. A. Salehi and C. A. Brackett, "Code division multiple-access techniques in optical fiber network-Part I: Fundamental principles," IEEE Trans. Commun., vol. 37, no. 8, pp. 824-833, Aug. 1989.

[8] J. A. Salehi, "Code division multiple-access techniques in optical fiber network-Part II: Systems performance analysis," IEEE Trans. Commun., vol. 37, no. 8, pp. 834-842, Aug. 1989.

[9] H. M. H. Shalaby, "Performance analysis of optical synchronous CDMA communication systems with PPM signaling," IEEE Trans. Commun., vol. 43, no. 2-4, pp. 624-634, Feb.-Apr. 1995

[10] H. M. H. Shalaby, "A performance analysis of optical overlapping PPMCDMA communication systems," J. Lightw. Technol., vol. 17, no. 3 pp. 426-433, Mar. 1999.

[11] M. H. M. Shalaby, "Direct detection optical overlapping PPM-CDMA communication systems with double optical hardlimiters," J. Lightw. Technol., vol. 17, no. 7, pp. 1158-1165, Jul. 1999.

[12] T. Ohtsuki, "Performance of multicode direct-detection optical CDMA systems," in Proc. IEEE Global Telecommun. Conf., 1998, vol. 6, pp. 3227-3232.
[13] S. V Maric, O. Moreno, and C. J. Corrada, "Multimedia transmission in fiber-optic LANs using optical CDMA," J. Lightw. Technol., vol. 14, no. 10, pp. 2149-2153, Oct.1996.

[14] W. C. Kwong and G.-C. Yang, "Multiple-length extended carrier-hopping prime codes for optical CDMA systems supporting multirate multimedia services," J. Lightw. Technol., vol. 23, no. 11, pp. 3653-3662, Nov. 2005 .

[15] G.-C. Yang, "Variable-weight optical orthogonal codes for CDMA network with multiple performance requirements," IEEE Trans. Commun., vol. 44, no. 1, pp. 47-55, Jan. 1996.

[16] B. M. Ghaffari, and J. A. Salehi, "Multiclass, multistage, and multilevel fiber-optic CDMA signaling techniques based on advanced binary optical logic gate elements," IEEE Trans. Commun., vol. 57, no. 5, pp. 1424-1432, May 2009.

[17] H. Beyranvnad, B. Ghaffari, and J. A. Salehi, "Multirate, differentiatedQoS, and multilevel fiber-optic CDMA system via optical logic gate elements," J. Lightw. Technol., vol. 27, no. 19, pp. 4348-4359, Oct. 2009

[18] C.-H. Chen, H.-Y. Chu, G.-C. Yang, C.-Y. Chang, and W. C. Kwong, "Performance analysis of double-weight optical CDMA under the samebit-power assumption," IEEE Trans. Commun., vol. 59, no. 5, pp. 12471252, May 2011

[19] L.-S. Chen, G.-C. Yang, C.-Y. Chang, and W. C. Kwong, "Study of power control on double-weight codes with an arbitrary maximum crosscorrelation value in variable-QoS optical CDMA," J. Lightw. Technol. vol. 29, no. 21, pp. 3293-3303, Nov. 2011.

[20] G.-C. Yang, C.-H. Chen, and W. C. Kwong, "Accurate analysis of doubleweight optical CDMA with power control," IEEE Trans. Commun., vol. 60, no. 2, pp. 322-327, Feb. 2012

[21] H. Beyranvand, S. A. Nezamalhosseini, J. A. Salehi, and F. Marvasti, "Performance analysis of equal-energy two-level OCDMA system using generalized optical orthogonal codes," J. Lightw. Technol., vol. 31, no. 10, pp. 1573-1584, May 2013.

[22] H.-H. Yuan, G.-C. Yang, C.-Y. Chang, and W. C. Kwong, "Accurate analysis of double-weight codes with an arbitrary maximum cross-correlation value for two-chip-power optical CDMA," IEEE Trans. Commun., vol. 61, no. 6, pp. 2488-2497, Jun. 2013.

[23] T. Miyazawa and I. Sasase, "Multi-rate and multi-BEP transmission scheme using adaptive overlapping pulse-position modulator and power controller in optical CDMA systems," J. Commun. Netw., vol. 7, no. 4, pp. 462-470, Dec. 2005

[24] A. G. Sabbagh and M. M. Kakhki, "Performance analysis of twolevel asynchronous optical CDMA systems utilizing wrapped OPPM,' J. Lightw. Technol., vol. 32, no. 1, pp. 122-129, Jan. 2014.

[25] F. R. K. Chung, J. A. Salehi, and V. K. Wei, "Optical orthogonal codes: Design, analysis, and applications," IEEE Trans. Inform. Theory, vol. 35, no. 3, pp. 595-604, May 1989

[26] C.-S. Brs, T. Banwell, I. Glesk, P. R. Prucnal, and W. C. Kwong, "Optical pulse position modulation processing: Architecture and demonstration in an optical code division multiple access system," J. Opt. Netw., vol. 5 , no. 12, pp. 915-926, Dec. 2006.

[27] H. M. H. Shalaby, "Optical OPPM-CDMA receivers with chip-level detectors," IEE Proc.-Commun., vol. 148, no. 1, pp. 31-37, Feb. 2001.

[28] A. G. Sabbagh and M. M. Kakhki, "Capacity analysis of asynchronous optical CDMA systems utilizing wrapped OPPM: multiple access interference limited case," J. Lightw. Technol., vol. 31, no. 15, pp. 2613-2620, Aug. 2013.

[29] M. Azizoglu, J. Salehi, and Y. Li, "Optical CDMA via temporal codes," IEEE Trans. Commun., vol. 40, no. 7, pp. 1162-1170, Jul. 1992

[30] R. M. Gagliardi and S. Karp, "Optical digital communications," in Optical Commun., 2nd ed. New York, NY, USA: Wiley, 1995, ch. 6, sec. 6, pp. 205-206.

Authors' biographies not available at the time of publication. 